\title{
An Efficient Method for Air Quality Evaluation via ANN-based Image Recognition
}

\author{
Xiaoguang Chen, Yaru Li and Dongyue Li \\ Department of Mathematics, College of Sciences, North China University of Techonology, Beijing 100141, P.R.China
}

\begin{abstract}
In recent years, air pollution problem has been the focus of public attention. In this paper, we proposed an efficient algorithm to evaluate the Air Quality Index (AQI) based on image recognition technology. In offline stage, some distinctive features extracted from the photos which are captured by common digital cameras, and then a prediction model of backpropagation neural network (BPNN) is trained. In online stage, the feature vectors extracted from the images are fed to the trained BPNN model to output the AQI value. Experimental results show that the proposed algorithm can produce the AQI evaluation with a considerable accuracy $93.78 \%$.
\end{abstract}

Keywords-Air Quality Index (AQI); image recognition; backpropagation neural network

\section{INTRODUCTION}

Air pollution is one aspect of the broader topic of environmental issues in China [1, 2]. Various forms of pollution have increased as China has industrialized, which has caused widespread environmental and health problems. On March 6, 2013, a user of SINA Weibo posted a microblog [3] with a composition of 225 photos, as shown in Figure I, which were captured at fixed location (East Third Ring, Beijing) and fixed time (8 - 9 AM) from March 1, 2012 to March 5, 2013. From Figure I, we can see that smoggy is the dominant air condition in these 225 days in Beijing. To monitor the situation of air pollution, the relevant departments should monitor the degree of air pollution. The most direct and traditional method for such purpose is to collect and analyze the air pollutants such as fine particulate matter, respirable particulate matter, sulfur dioxide, nitrogen dioxide, ozone, carbon monoxide and the others pollutants [1]. Obviously, the chemical methods need a much expensive cost and very complex procedure.

In recent years, researchers developed some novel methods for air quality evaluation such as utilizing visual information hidden in the scene photos [3-10]. Liu et al. [5, 6] proposed a new method for air quality evaluation method, which only uses the surveillance image. They firstly build different time series data with different image quality metrics, i.e. the noise brightness ratio, the contrast level and the blur level. Then a Gompertz type diffusion process (GTDP) of stochastic growth and the detrend fluctuation analysis (DFA) method are utilized to analyze and fit the trend of these series. Finally, the parameters of the GTDP model and the DFA model are used to evaluate and classify the air quality associated with the image quality change level of different atmospheres. In [7, 8], an internet surveillance camera was used to quantify air quality with an algorithm based on the regression analysis of the relationship between measured reflectance components from a surface material and the atmosphere. In [9], the fractal characteristics of the images obtained by CCD image sensor are analyzed to recognize the suspended particles in the atmosphere for air pollution research.

Inspired by the related works and considering human visual perception, we designed a simple but efficient algorithm for air quality evaluation from daily photos captured by mobile phones or digital cameras. In the proposed algorithm, the histogram features and edge extraction of the input image are mainly considered, and then a model of back-propagation neural network (BPNN) is trained with the collected data comprising of photos and AQI values. While in evaluation stage, the BPNN model takes the feature vector computed from the image as input, and conducts forward computation to produce the AQI prediction.

The rest of this paper is organized as follows. Section 2 introduces the proposed algorithm in detail, including image preprocessing, image feature extraction, BPNN model design and AQI prediction; Section 3 demonstrates the experimental results and analysis; Conclusion and future works are given in Section 4.

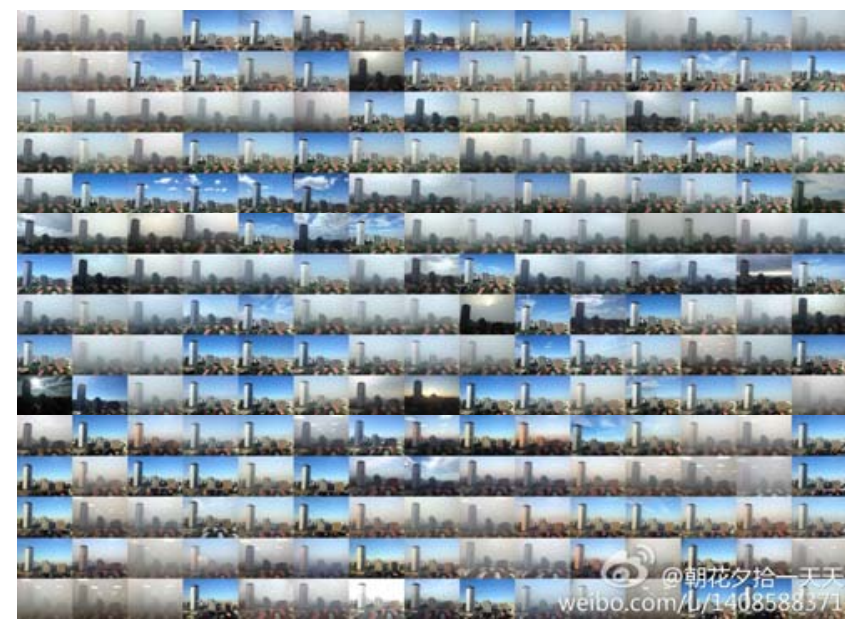

FIGURE I. PHOTO COLLECTION: "SKY IN BEIJING” 


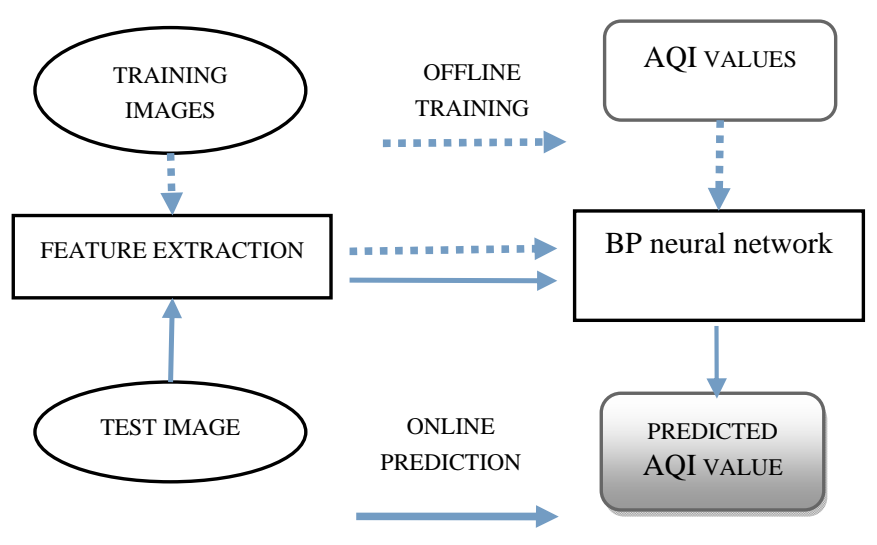

FIGURE II. THE FLOWCHART OF PROPOSED METHOD FOR IMAGE-

\section{PROPOSED METHOD}

The flowchart of proposed method is illustrated in Figure II, which consists of two phases: offline model training and online AQI prediction. We will describe the detail of each procedure in the following subsections.

\section{A. Image Feature Extraction}

Generally, feature extraction is a key step for most image recognition algorithms. To imitate the ability of human visual perception, we choose some typical images with different AQI values to observe, as shown in Figure III. Given close observation, we find that the following results. Due to serious diffuse reflection caused by the large number of particles, the images with high AQI are much misty and bright, and the structural objects are blurred without clear boundaries. On the contrary, due to good air quality and light environment, the images with low AQI are much clear and have uniform brightness, and the objects also have clear structures. Therefore, we are inspired to evaluation the AQI based on the proportions of bright pixels and the edge pixels in the whole image.
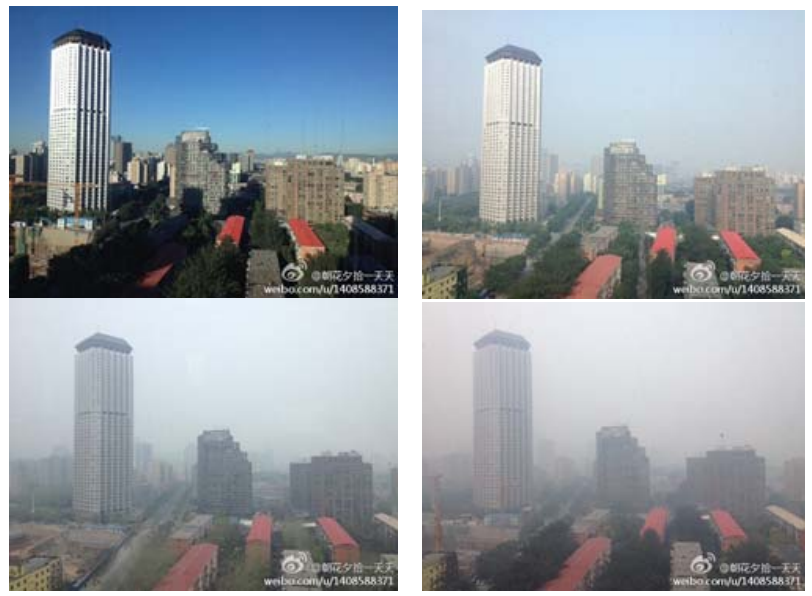

FIGURE III. IMAGES WITH DIFFERENT AQI VALUES 10, 125, 205 AND 280
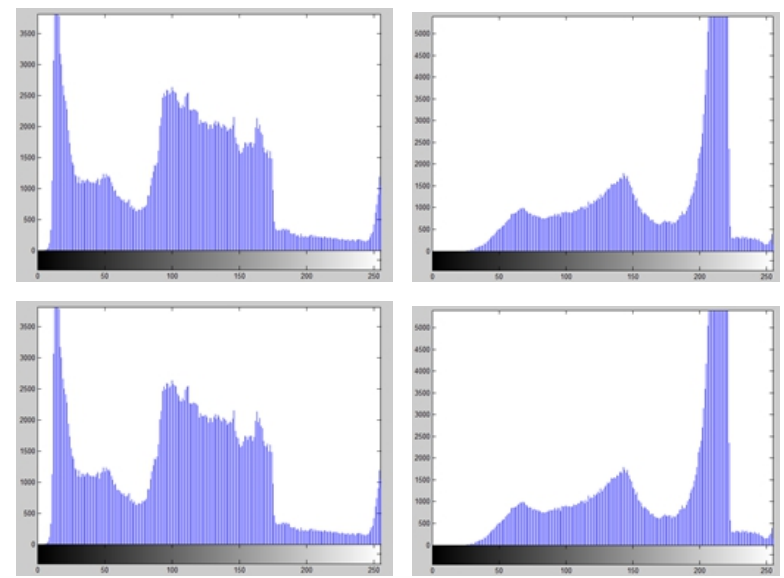

FIGURE IV. THE BRIGHTNESS HISTOGRAMS FOR THE IMAGES IN FIGURE III WITH AQI 10, 125, 205 AND 280 RESPECTIVELY

(1) Ratio of high-brightness pixels

For an image, color conversion from RGB to $\mathrm{YCbCr}$ is carried out, and therefore the Y-channel can represent the brightness of each pixel in the image. Then the histogram of the Y-channel is computed as the first feature for proposed algorithm. Figure IV illustrates the histograms of brightness computed for the four images in Figure III. The brightness histogram with different AQI have different distribution obviously. A lower AQI will result in a more uniform the distribution of the corresponding brightness histogram, and a higher AQI will result in a non-uniform distribution with a concentration in the right high-brightness interval.

For simplicity, the ratio of high-brightness pixels is computed by following steps: (1) Perform RGB $\rightarrow$ YCbCr color conversion; (2) Count the number of pixels whose $\mathrm{Y}$ (brightness) value is larger than 128; (3) Compute the ratio between the high-brightness pixels to the overall pixels in the image. The ratio tends to increase as the AQI value increases and it has a value interval $[0,1]$.
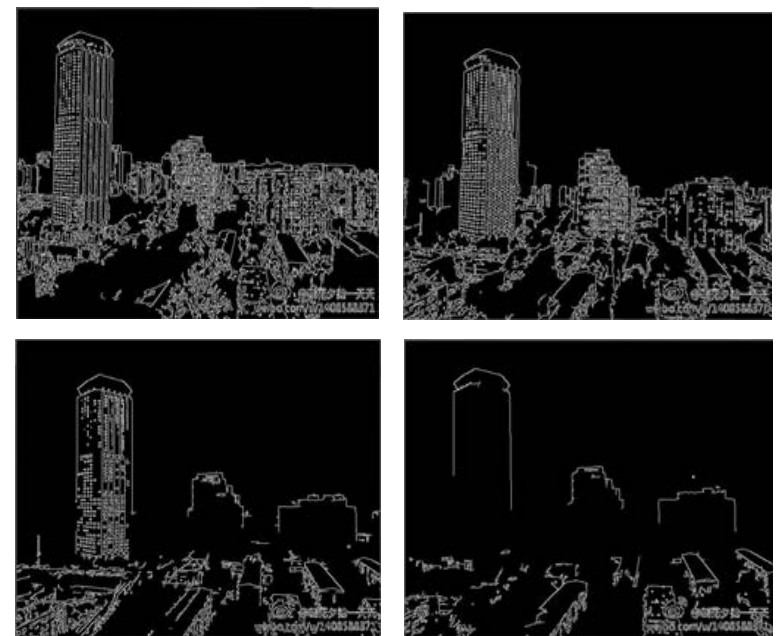

FIGURE V. THE RESULTS OF CANNY EDGE DETECTION FOR THE IMAGES IN FIGURE 3 WITH AQI 10, 125, 205 AND 280 RESPECTIVELY 


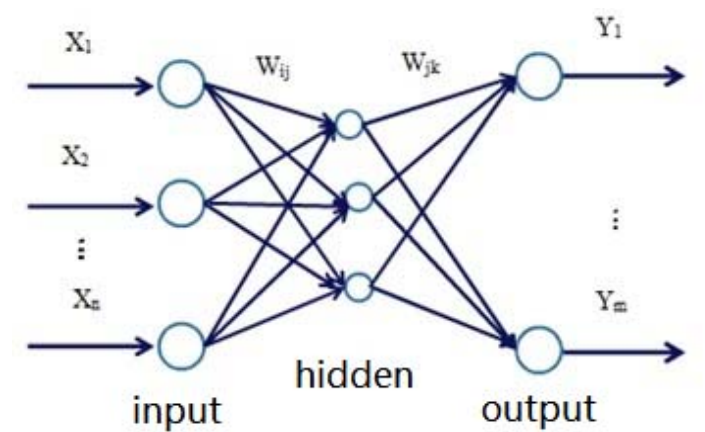

FIGURE VI. ILLUSTRATION OF BPNN WITH THREE-LAYER STRUCTURE

\section{(2) Ratio of edge points}

The edge detection can be applied to extract the boundaries of the structural objects in the image. The results of Canny edge detection [11] for the images in Figure III are given in Figure V. Obviously, the number of edge points is decreased gradually while AQI value increases.

The ratio of edge points is computed by following steps: (1) Conduct edge detection with Canny operator; (2) Count the number of detected edge points; (3) Compute the ratio between the numbers of edge points and overall pixels in image. By definition, the range of the ratio is $[0,1]$.

\section{B. Back-Propagation Neural Network}

Artificial neural networks (ANNs) has a wide application in many fields such as image recognition $[12,13]$. The diagram of three-layer BPNN is depicted in Figure VI. The learning processing of BP neural network is comprised of the forward propagation of input information and the back propagation of error. In the training stage, the network adjusts the weights ( $W$ in Fig.6) which connect the neural units in different layers according the error to the input ( $X$ in Fig. 6$)$ and output ( $Y$ in Fig. 6) information of different samples. We refer the reader to the related literatures for more knowledge about ANN.

The proposed algorithm uses a three-layer BPNN with 2, 10 and 1 neurons for the input, hidden and output layers respectively. Despite of the simple network structure, it achieved an excellent experimental performance as described in the next section.

\section{EXPERIMENTAL RESULTS AND ANALYSIS}

\section{A. Data Preparation}

For the experimental evaluation, we collect the sample images from SINA Weibo [3] and select some meaningful data by follows steps. (1) Choose the photos which were captured at same location and at similar time from March 2013 to March 2014. (2) Drop out the photos which were captured in snowy and rainy days. (3) Leave only one photo for the same AQI for reducing processing time. (4) Query the AQI values from office websites [2]. Finally, 41 images are selected for the experiments, and the training/testing sets consist of 34/7 images respectively, as shown in Figure VII and Figure VIII.
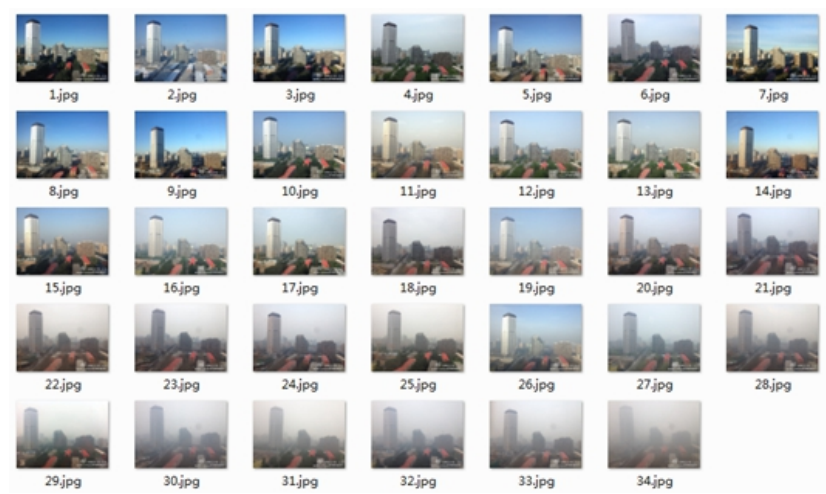

FIGURE VII. IMAGES IN TRAINING SET
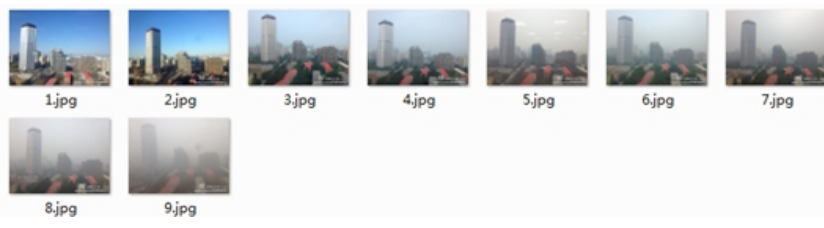

FIGUREVIII. IMAGES IN TESTING SET

To avoid the magnitude difference of the data, the AQI value is scaled down by $1 / 500$ in training phase and is scaled up by 500 in the testing phase.

\section{B. Results and Analysis}

To validate the effectiveness of proposed algorithm, we compute the relative error for each testing image, and present the predicted air quality level (AQL) simultaneously. Table I reports the predicted AQI and AQL values and prediction errors. As listed, the results show that our algorithm produced an average error $6.22 \%$ in the aspect of AQI prediction, and achieved an accuracy $100 \%$ for AQL prediction.

By comparing the prediction error with actual AQI values, we find that the prediction of sample images with index 5, 6,7 and 8 have smaller errors $(<5.5 \%)$, but the ones with index 1,2 , 3,4 and 9 have larger errors $(>5.5 \%)$. Considering the training manner of BPNN, this phenomenon may be caused by the nonuniform distribution of the AQI value in the training data set. In addition, due to the various imaging condition, each image exhibits different characteristic and quality which leads to various reliability of feature extraction for both of training and testing purpose.

\section{CONCLUSION}

In this paper, a new method for air quality index prediction and air quality assessment based on image recognition technology is proposed. Based on the theory of pattern recognition, the algorithm builds a model of BP neural network to predict AQI from a daily photo captured by common imaging devices such as mobile phone and digital cameras. Experimental results show a passable prediction accuracy 93.78\%. Compared with traditional AQI evaluation method, the proposed method not only eliminates the complicated process of collecting and analyzing air pollutants, but also saves the expensive cost. 
TABLE I. EXPERIMENTAL RESULTS ON TESTING SET

\begin{tabular}{cccccc}
\hline $\begin{array}{c}\text { Sample } \\
\text { Index }\end{array}$ & Actual AQI & Actual AQL & Predicted AQI & Predicted AQL & Prediction Error of AQI \# \\
\hline 1 & 35 & Excellent & 31.83 & Excellent & $9.06 \%$ \\
\hline 2 & 85 & Good & 91.45 & Good & $7.59 \%$ \\
\hline 3 & 90 & Good & 77.55 & Good & $13.83 \%$ \\
\hline 4 & 135 & Lightly polluted & 145.91 & Lightly polluted & $5.08 \%$ \\
\hline 5 & 185 & Moderately polluted & 175.59 & Moderately polluted & $3.61 \%$ \\
\hline 6 & 195 & Moderately polluted & 187.96 & Moderately polluted & $1.70 \%$ \\
\hline 7 & 205 & Heavily polluted & 208.48 & Heavily polluted & $1.37 \%$ \\
\hline 8 & 270 & Heavily polluted & 273.70 & Heavily polluted & $5.65 \%$ \\
\hline 9 & 330 & Severely polluted & 311.37 & Severely polluted & $\mathbf{6 . 2 2 \%}$ \\
\hline
\end{tabular}

\section{REFERENCES}

[1] https://en.wikipedia.org/wiki/Air_pollution.

[2] Beijing Air Report, http://www.beijing-air.com/.

[3] microblog page: http://weibo.com/u/1408588371.

[4] X. Fan, H. Wu, "The application of image recognition in monitoring of air quality," Henan Sciences, 2013, vol.31, no.7, pp.1004-1007.

[5] H. Liu, F. Li, F. Xu, H. Lu, "The evaluation of air quality using image quality," Chinese Journal of Image and Graphics, 2011, vol.16, no.6, pp.1030-1037

[6] H. Liu, H. Lu. "Imaging air quality evaluation based on Noise Brightness Ratio \& Gompertz Type Diffusion Process," In Proc. of Congress on Image and Signal Processing (CISP), 2010, pp.2332-2335.

[7] C. J. Wong, M. Z. Matjjafri, K. Abdullah, H. S. Lim, K. L. Low. "Temporal air quality monitoring using surveillance camera," In Proc. of IEEE International Symposium on Geoscience and Remote Sensing (IGARSS), 2007, pp.2864-2868

[8] C. J. Wong, M. Z. Matjafri, K. Abdullah, H. S. Lim, "Temporal and spatial air quality monitoring using internet surveillance camera and ALOS satellite image," Aerospace Conference, 2009, pp.7336:1-7.

[9] D. Wang, "Image recognition of atmospheric suspended particles based on Fractal Theory," Wuhan University of Technology, 2006.

[10] Y. Inoue, A. Kaga, K. Yamaguchi, S. Kamoi, "Development of an automatic system for counting asbestos fibers using image processing," Particulate Science and Technology, 2007, vol.16, no.4, pp.263-279.

[11] J. Canny, "A computational approach to edge detection," IEEE Trans. Pattern Analysis and Machine Intelligence, 1986, vol.8, no.6, pp.679698.

[12] J. Xu, K. Yamada, K. Seikiya, "Effect of different features to drill-wear prediction with back propagation neural network," Precision Engineering, 2014, vol.38, no.4, pp.791-798

[13] W. Wang, B. Yu, "Text categorization based on combination of modified back propagation neural network and latent semantic analysis," Neural Computing and Applications, 2009, vol.18, no.8, pp.875-881. 